\title{
Dissection of subclonal evolution by temporal mutation profiling in chronic lymphocytic leukemia patients treated with ibrutinib
}

\author{
Ambrus Gángó ${ }^{1 *}$, Donát Alpár ${ }^{1 *}$, Bence Galik ${ }^{2,3 *}$, Dóra Marosvári ${ }^{1}$, Richárd Kiss ${ }^{1}$, Viktória Fésüs ${ }^{1}$, Dóra Aczél ${ }^{1}$, \\ Ediz Eyüpoglu ${ }^{1}$, Noémi Nagy ${ }^{1}$, Ákos Nagy ${ }^{1}$, Szilvia Krizsán ${ }^{1}$, Lilla Reiniger ${ }^{1}$, Péter Farkas ${ }^{4}$, András Kozma ${ }^{5}$, Emma Ádám ${ }^{5}$, \\ Szabolcs Tasnády ${ }^{5}$, Marienn Réti ${ }^{5}$, András Matolcsy ${ }^{1}$, Attila Gyenesei ${ }^{2,3}$, Zoltán Mátrai ${ }^{5 \dagger}$ and Csaba Bödör (D) ${ }^{1 \dagger}$ \\ ${ }^{1}$ MTA-SE Momentum Molecular Oncohematology Research Group, 1st Department of Pathology and Experimental Cancer Research, Semmelweis \\ University, Budapest, Hungary \\ ${ }^{2}$ Bioinformatics Research Group, Bioinformatics and Sequencing Core Facilities, Szentágothai Research Centre, University of Pécs, Pécs, Hungary \\ ${ }^{3}$ Department of Clinical Molecular Biology, Medical University of Bialystok, Białystok, Poland \\ ${ }^{4}$ 3rd Department of Internal Medicine, Semmelweis University, Budapest, Hungary \\ ${ }^{5}$ Department of Haematology and Stem Cell Transplantation, St. István and St. László Hospital, Budapest, Hungary
}

The Bruton's tyrosine kinase (BTK) inhibitor ibrutinib is inducing durable responses in chronic lymphocytic leukemia (CLL) patients with refractory/relapsed disease or with TP53 defect, with BTK and phospholipase C gamma 2 (PLCG2) mutations representing the predominant mechanisms conferring secondary ibrutinib resistance. To understand the landscape of genomic changes and the dynamics of subclonal architecture associated with ibrutinib treatment, an ultra-deep next-generation sequencing analysis of 30 recurrently mutated genes was performed on sequential samples of 20 patients, collected before and during single-agent ibrutinib treatment. Mutations in the SF3B1, MGA and BIRC3 genes were enriched during ibrutinib treatment, while aberrations in the BTK, PLCG2, RIPK1, NFKBIE and XPO1 genes were exclusively detected in posttreatment samples. Besides the canonical mutations, four novel BTK mutations and three previously unreported PLCG2 variants were identified. BTK and PLCG2 mutations were backtracked in five patients using digital droplet PCR and were detectable on average 10.5 months before clinical relapse. With a median follow-up time of 36.5 months, $7 / 9$ patients harboring BTK mutations showed disease progression based on clinical and/or laboratory features. In conclusion, subclonal heterogeneity, dynamic clonal selection and various patterns of clonal variegation were identified with novel resistance-associated BTK mutations in individual patients treated with ibrutinib.

Key words: chronic lymphocytic leukemia, ibrutinib, clonal evolution, targeted therapy, precision medicine

Abbreviations: BCR: B cell receptor; BTK: Bruton's tyrosine kinase; CLL: chronic lymphocytic leukemia; CME: convergent mutation evolution; ddPCR: digital droplet PCR; NGS: next-generation sequencing; PBMC: peripheral blood mononuclear cells; PLCG2: phospholipase C Gamma 2; SNV: single nucleotide variant; VAF: variant allele frequency

Additional Supporting Information may be found in the online version of this article.

Conflict of interest: The authors declare no potential conflicts of interest.

Grant sponsor: Emberi Eroforrások Minisztériuma; Grant numbers: EFOP-3.6.3-VEKOP-16-2017-00009, ÚNKP-18-3-I-SE-48, ÚNKP18-4-SE-62; Grant sponsor: Higher Education Institutional Excellence Programme within the framework of the Molecular Biology thematic programme of the Semmelweis University; Grant sponsor: Magyar Tudományos Akadémia; Grant numbers: János Bolyai Research Scholarship Program (BO/00320/18/5), LP-95021; Grant sponsor: Nemzeti Kutatási, Fejlesztési és Innovációs Hivatal (Hungarian National Research, Development and Innovation Office); Grant numbers: KH17-126718, K_16\#119950, NVKP_16-1-2016-0004, NVKP_16-12016-0005

*A.G., D.A. and B.G. contributed equally to this work

${ }^{\dagger}$ Z.M. and C.B. shared joint senior authorship

DOI: $10.1002 /$ ijc.32502

This is an open access article under the terms of the Creative Commons Attribution-NonCommercial License, which permits use, distribution and reproduction in any medium, provided the original work is properly cited and is not used for commercial purposes.

History: Received 4 Jan 2019; Accepted 28 May 2019; Online 10 Jun 2019

Correspondence to: Dr. Csaba Bödör, MTA-SE Momentum Molecular Oncohematology Research Group, 1st Department of Pathology and Experimental Cancer Research, Semmelweis University, Üllői út 26, 1085 Budapest, Hungary, Tel.: +36-1-215-7300, Fax: +36-1-317-1074,

E-mail: bodor.csaba1@med.semmelweis-univ.hu 


\section{What's new?}

Although the Bruton's tyrosine kinase (BTK) inhibitor, ibrutinib has revolutionized the treatment of chronic lymphocytic leukemia, $20 \%$ of patients still show disease progression. Comprehensive characterisation of mechanisms underlying ibrutinib resistance and the related changes in the subclonal architecture induced by the selective pressure of the treatment may usher in new clinical advances. This time-resolved ultra-deep genomic scrutiny of mutation target genes reveals unique patterns of highly dynamic clonal variegation associated with BTK inhibition and identifies novel resistance-associated BTK mutations in individual patients. Furthermore, evidence suggests that sensitive molecular monitoring of treatment response can facilitate the early detection of impending relapse.

\section{Introduction}

Chronic lymphocytic leukemia (CLL) is characterized by substantial clinical and genetic heterogeneity. Recent whole-exome and whole-genome sequencing studies unraveled recurrently mutated driver genes in CLL, including ATM, NOTCH1, SF3B1, BIRC3, NKFBIE, MYD88 and TP53, and identified clonal evolution as the major mechanism driving disease progression in the context of standard chemo-immunotherapy. ${ }^{1-7}$ Patients with TP53 aberrations have typically been characterized by refractoriness to standard therapies and particularly poor outcome with rapid selection of the resistant clones during standard therapies. ${ }^{8,9}$

The irreversible Bruton's tyrosine kinase (BTK) inhibitor, ibrutinib has been changing the treatment paradigm of CLL with remarkable outcomes in first line as well as in relapsed CLL, including high-risk patients with $17 \mathrm{p}$ deletion and/or TP53 mutation. ${ }^{10-13}$ Despite the durable responses observed in the majority of patients, approximately $20 \%$ of them develop resistance, with mutations in the BTK and phospholipase $\mathrm{C}$ gamma 2 (PLCG2) genes representing the predominant mechanisms conferring secondary ibrutinib resistance. ${ }^{14,15}$ The loss of function BTK Cys481 mutations leading to impaired ibrutinib binding and/or the gain of function PLCG2 Arg665Trp/Ser707Tyr/Leu845Phe mutations resulting in continuous B-cell receptor (BCR) signaling can be detected in the vast majority of ibrutinib resistant patients. ${ }^{16-18}$ Based on initial studies, these mutations are commonly present in multiple independent subclones suggesting parallel clonal evolution and their emergence predates clinical progression and relapse. ${ }^{16,18}$ Indeed, clonal shifts identified by whole-exome sequencing during the early periods of ibrutinib treatment in one-third of the patients were associated with disease progression, reflecting great evolutionary capacity potentially leading to emergence of drug-resistant clones. ${ }^{19}$ Given the abysmal outcome of patients discontinuing ibrutinib, comprehensive characterization of mechanisms underlying ibrutinib resistance and the related changes in the subclonal architecture induced by the selective pressure of the treatment have major clinical importance and represent an urgent unmet need. ${ }^{20}$

To dissect the clonal evolution affecting all relevant mutation targets in CLL in the context of ibrutinib therapy, we performed a temporal mutation profiling by analyzing 30 genes in paired pretreatment and posttreatment samples of CLL patients receiving ibrutinib therapy outside the setting of a clinical trial.

\section{Materials and Methods \\ Patient samples}

Sequential samples from 20 consecutive patients (12 males and 8 females), with a median age of 63 years treated with singleagent ibrutinib were included in our study with clinical characteristics listed in Supporting Information Table S1. Our cohort represented a pretreated patient group with a median of 2 (range: 1-5) lines of prior therapies. These patients were initially granted access to ibrutinib via a case by case individual application process available in Hungary since July 2014, with ibrutinib fully supported by the National Health Insurance Fund from 2017. Pretreatment peripheral blood mononuclear cells (PBMC) were available from all patients, with matching posttreatment samples as outlined in Figure 1a. The IGHV mutation status was determined according to the most recent European Research Initiative on CLL recommendations ${ }^{21}$ with deletions 13q, 11q and 17p, as well as trisomy 12 analyzed by interphase fluorescence in situ hybridization using Vysis probe sets (Abbott Molecular, Lake Bluff, IL). The proportion of CLL cells in the samples was assessed by flow cytometry using CD5/CD19/ CD23/CD45 staining. PBMC-derived DNA samples from five healthy volunteers were used as negative controls. Written informed consent from all patients was obtained for the study which was conducted in accordance with the Declaration of Helsinki and approved by the Hungarian Medical Research Council.

\section{Customized ultradeep next-generation sequencing}

Targeted ultra-deep next-generation sequencing (NGS) analysis of 30 recurrently mutated genes, including $A T M, B C O R, B I R C 3$, BRAF, BTK, CHD2, DDX3X, EGR2, EIF2A, EP300, FBXW7, HIST1H1E, IGLL5, KLHL6, KMT2D, LRP1B, MED12, MGA, MYD88, NFKBIE, NOTCH1, PLCG2, POT1, RIPK1, RPS15, SAMHD1, SF3B1, TP53, XPO1 and ZMYM3 (Supporting Information Table S2) with a published frequency of $\geq 2 \%^{1,2,4-7}$ was performed using the TruSeq Custom Amplicon approach (Illumina, San Diego, CA) with maximum input of genomic DNA samples extracted from PBMCs. After quality control and equimolar pooling, libraries were sequenced on a HiSeq 4000 Instrument (Illumina) using $150 \mathrm{bp}$ paired-end chemistry. The variant allele frequencies (VAF) were normalized considering the proportion of CLL cells as determined by flow cytometry. The median follow-up time at the time of the NGS analysis was 22.5 months (range: 3-34 months). 
(a)
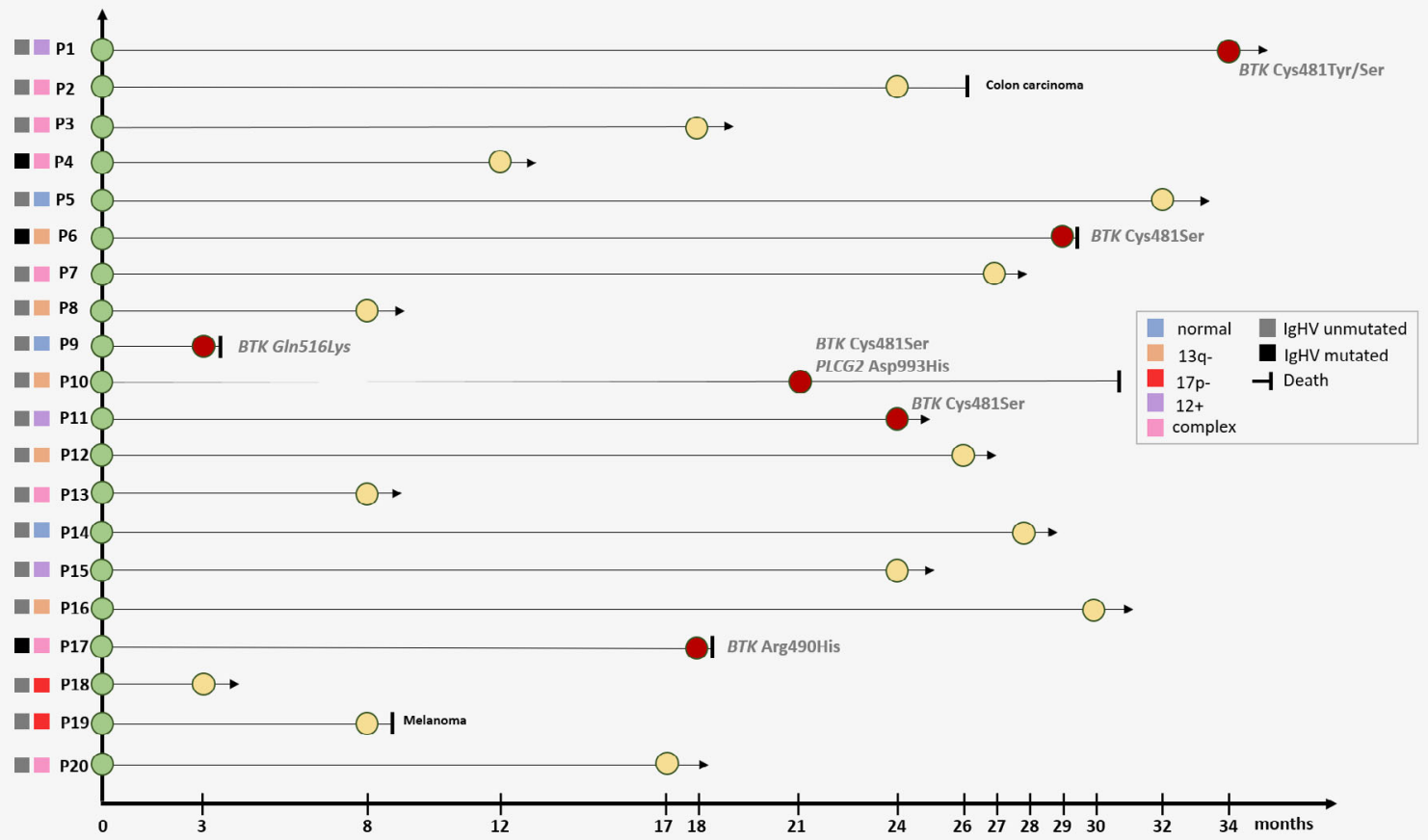

(b)

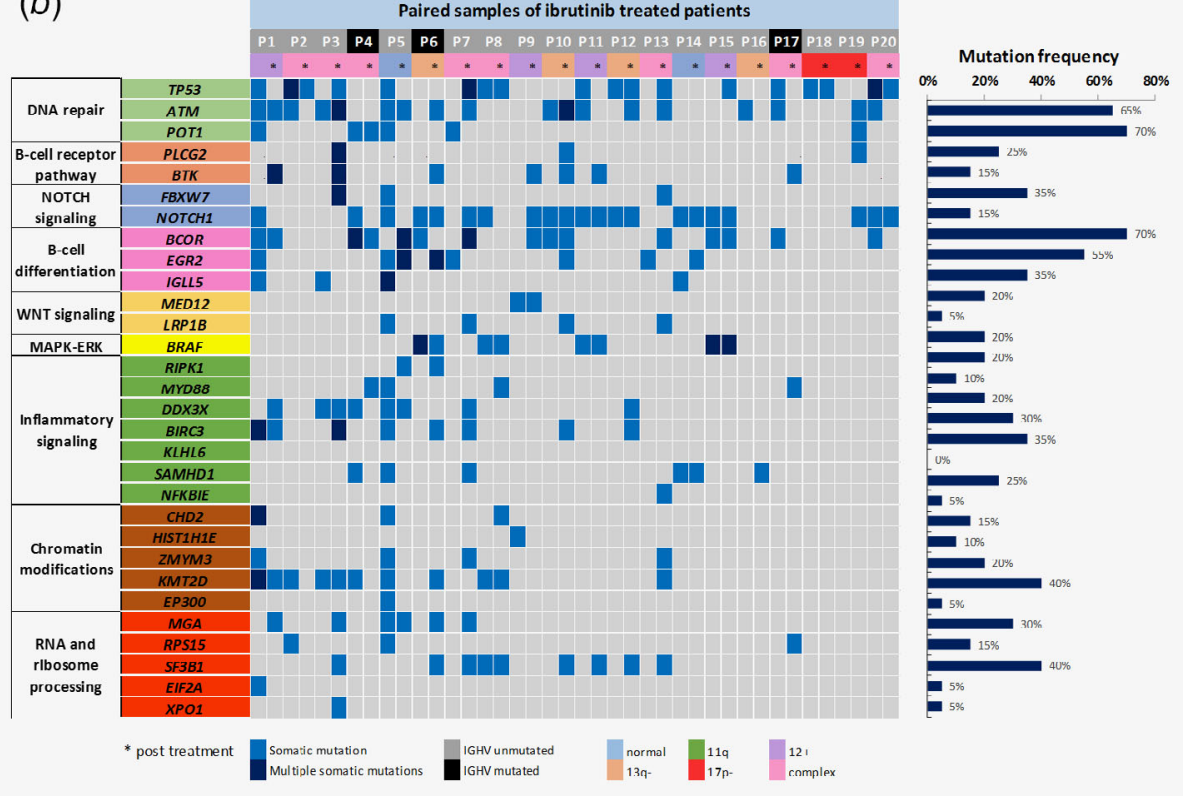

(c) Temporal distribution of mutations

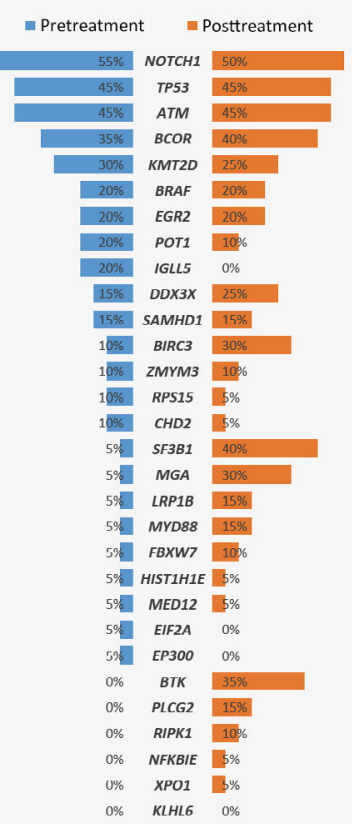

Figure 1. (a) Timeline and basic cytogenetic features of the 20 patients treated with ibrutinib. Red circles denote patients who progressed on ibrutinib with a BTK or PLCG2 mutation as determined by next-generation sequencing. (b) Heat map displaying the somatic variants detected in the 30 target genes analyzed in the sequential samples of 20 patients treated with ibrutinib. Illustrated are the distribution of the somatic variants, mutation status of the IGHV gene segment, cytogenetic profile as determined by fluorescence in situ hybridization, as well as the mutation frequency of the individual genes for all cases. (c) Comparison of the mutation frequency in the 30 genes analyzed between the pretreatment and posttreatment specimens. (d) Different patterns of clonal composition and dynamics in four selected patients with TP53 mutations. Convergent mutation evolution was frequently observed with acquisition of multiple mutations within a gene, exemplified by multiple TP53 mutations in Patients \#2 and \#20. Interestingly, TP53 mutations showed a similar tendency to undergo clonal expansion or elimination under ibrutinib therapy. 

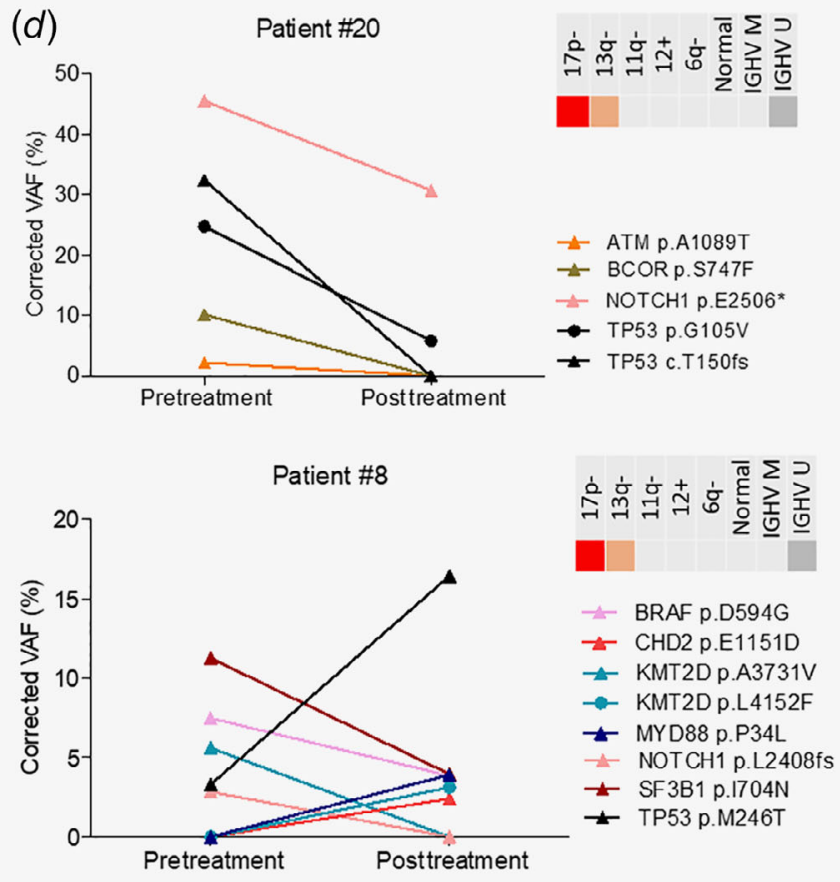

Figure 1. Continued

\section{Bioinformatics workflow}

During data preprocessing, sequencing reads were mapped to the Homo sapiens GRCh37 genome build using BWA v0.7.13 aligner from the BaseSpace Sequence Hub. BAM files were sorted and indexed by SAMtools v1.7 module and GATK v4.0 tool. Base Quality Score Recalibration was run on each sample to detect and correct systematic sequencing errors. Single nucleotide variant (SNV) and INDEL calling were performed with LoFreq v2.1 variant-caller ${ }^{22}$ that considers all dataset features, also including base-call qualities, mapping problems or base/INDEL misalignments, which are commonly ignored by other methods or only used for filtering. Assignment of each detected variant with a $p$ value allowed for a rigorous control of false positive findings. Raw variants, detected by LoFreq v2.1, were functionally annotated using SnpEff v4.3i as well as ANNOVAR v2017Jul17 tools, with the latter one also including up-to-date information from COSMIC, avSNP and CLINVAR databases. ${ }^{23,24}$ In addition, variants in the TP53 coding region were annotated using the TP53-specific Seshat and IARC databases. ${ }^{25,26}$ The raw sequencing data was uploaded to the European Nucleotide Archive (https:/www.ebi.ac.uk/ena, Primary Accession: PRJEB32120, Secondary Accession: ERP114759).

\section{Validation of somatic variants}

Bidirectional Sanger sequencing was applied to validate all somatic variants with a VAF of $>20 \%$ (Supporting Information Table S3) using primers listed in Supporting Information Table S4 with the detailed results presented in Supporting Information Figure S1. BTK Cys481Ser and PLCG2 Asp993His
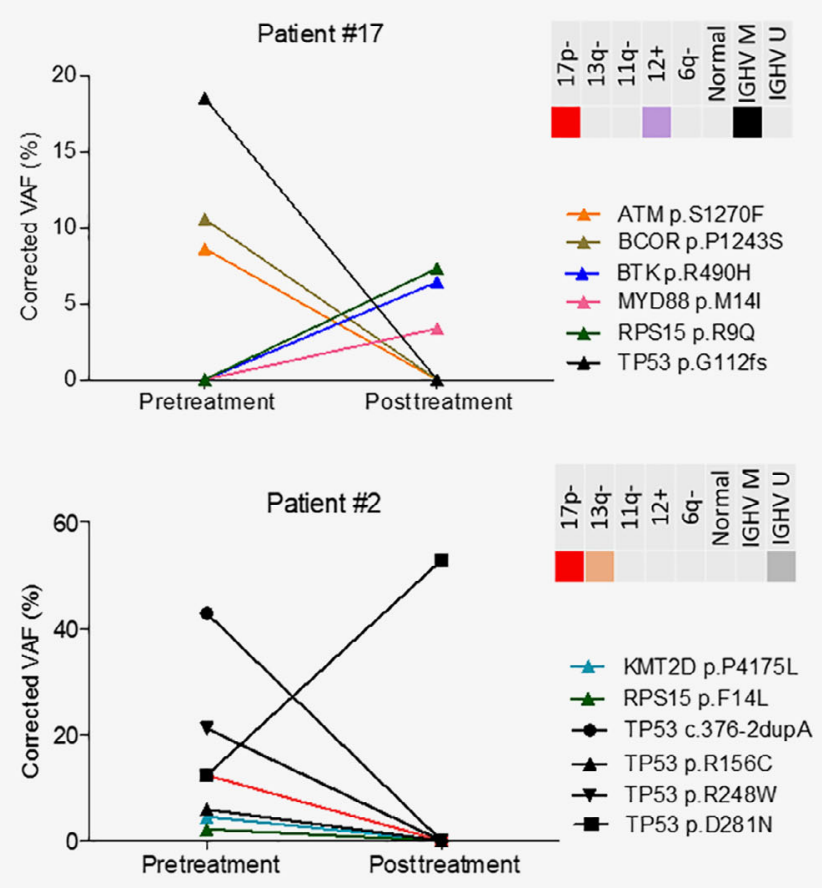

mutations were validated by droplet digital PCR (ddPCR; Supporting Information Fig. S2). Reactions were performed with $50 \mathrm{ng}$ input DNA using locus-specific assays for the wild type and mutant targets (Supporting Information Table S5) following the manufacturer's protocol. Droplets were created by the QX200 Automated Droplet Generator and reading was completed with the QX200 ddPCR system (Bio-Rad, Hercules, CA). Results were analyzed using the Bio-Rad QuantaSoft software. The $B T K^{\text {mut }}$ or $P L C G 2^{\text {mut }}$ allelic burden was determined as fractional abundance (FA) based on the percentage ratio between the number of mutant DNA molecules $(a)$ and the number of mutant $(a)$ plus wild-type $(b)$ molecules detected $[\mathrm{FA}=a /(a+b)]$.

\section{Results}

\section{Mutational landscape of the ibrutinib treated cohort}

The ultra-deep NGS revealed a total of 211 somatic variants in the 20 paired samples with an average allelic depth of 7,500x across the 30 genes analyzed (Fig. 1b, Supporting Information Table S6). The majority (157/211) of the variants represented subclonal alterations with VAF of $<10 \%$ (Supporting Information Fig. S3). A remarkable subclonal heterogeneity was detected across the cases with an average of five mutations (range: $0-19$ ) detected in individual patients, affecting an average of four genes (range: 0-18; Supporting Information Fig. S4). NOTCH1 (70\%, $14 / 20), \operatorname{ATM}(70 \%, 14 / 20), \operatorname{TP} 53(65 \%, 13 / 20)$ and BCOR $(55 \%$, $11 / 20$ ) represented the most frequently mutated target genes (Fig. 1b). All somatic variants with a VAF of $>20 \%$ were successfully validated by Sanger sequencing. 


\section{Temporal dissection of the mutational landscape}

The posttreatment samples carried a slightly higher number of variants compared to the pretreatment samples (118 vs. 93), with an average of 5.9 mutations (range: 1-16) in the posttreatment specimens and on average 4.7 mutations in the pretreatment samples (range: 0-19; Supporting Information Fig. S4). Temporally, NOTCH1, ATM, TP53 and BCOR represented the top four mutated target genes at baseline as well as posttreatment (Fig. 1c). While IGLL5, EIF2A and EP300 mutations were eliminated from the posttreatment samples, we observed an enrichment of SF3B1 (5\% vs. 40\%), MGA (5\% vs. $30 \%)$, BIRC3 (10\% vs. 30\%) mutations in the posttreatment samples compared to the pretreatment specimens (Fig. 1c), with a three-fold increase in mutation frequencies of MYD88 and $L R P 1 B$ genes after ibrutinib treatment (Fig. 1c). BTK, PLCG2, RIPK1, NFKBIE and XPO1 mutations were exclusively detected in the posttreatment samples in $35,15,10,5$ and $5 \%$ of the patients, respectively (Fig. 1c).

Various combinations of mutations were identified in all patients (Supporting Information Fig. S5). Convergent mutation evolution (CME) defined as acquisition of multiple mutations in the same gene ${ }^{27}$ was identified in $40 \%(12 / 30)$ of the genes analyzed, with 2-4 mutations detected within a particular target gene (Fig. 1b). Overall, CME was observed in 50\% (10/20) of patients, with this phenomenon documented in both pretreatment and posttreatment samples in four patients and in either pretreatment or posttreatment samples of three patients.

\section{Subclonal dynamics of TP53, BTK and PLCG2 mutations}

Mutations in the BTK or PLCG2 genes previously found to be associated with ibrutinib resistance were detected in $40 \%(8 / 20)$ and $5 \%(1 / 20)$ of the patients, respectively, with mutations exclusively detected in the posttreatment samples (Figs. $1 b$ and $1 c$ ). The BTK and PLCG2 variants co-occurred in $2 / 8$ patients carrying mutations in one of these genes with 1-4 variants present in individual patients (Supporting Information Table S6). In addition to the canonical BTK Cys481 and PLCG2 Asp993 hotspots, four novel BTK mutations at residues Arg28, Gly164, Arg490 and Gln516 were identified (Fig. $2 a$ ), with three previously unreported PLCG2 mutations at Phe82, Arg694 and Ser1192 (Fig. 2b), affecting altogether four different patients. Changes in the mutational composition and heterogeneity between the pretreatment and posttreatment samples of patients progressing on ibrutinib with BTK/PLCG2 mutations are illustrated in Supporting Information.

Of special interest were the frequent multiple TP53 mutations characterized by either elimination or expansion of the mutant clone, or elimination of the TP53 mutant subclone and concurrent emergence of an independent subclone with TP53 mutation(s) in the posttreatment sample, as illustrated by Patients \#20, \#17, \#8 and \#2, respectively (Fig. 1d). Notably, an alternating dynamics of BTK and TP53 mutations was observed in almost all patients carrying both alterations in any of their samples. The emergence of BTK mutations upon ibrutinib treatment was accompanied by the concurrent decrease of TP53 mutational abundance. Among the six patients harboring BTK Cys481 mutations (Patients \#1, \#5, \#6, \#10, \#11 and \#20), all four patients carrying TP53 mutations (Patients \#1, \#5, \#11 and \#20) demonstrated clonal elimination or reduction of the TP53 alteration in the posttreatment sample. Elimination of a TP53 mutation was also observed in Patient \#17, acquiring a noncanonical $B T K$ mutation upon ibrutinib treatment. On the other hand, subclones carrying TP53 mutations persisted or expanded in 8/20 patients (Patients \#2, \#3, \#7, \#8, \#12, \#13, \#15 and \#18), among whom only Patient \#3 carried two previously unknown BTK mutations with currently ambiguous clinical significance. Indeed, canonical BTK Cys481 mutations were never observed in patients with detectable TP53 mutation in the posttreatment sample.

\section{Clinical outcome and follow-up of patients}

Clinically, with an extended median follow up time of 36.5 months (range: 3-43 months) documented after performing the initial NGS analysis, 65\% (13/20) of patients remain on ibrutinib with at least partial or complete remission. Three patients (Patients \#9, \#10 and \#17) underwent Richter's transformation and succumbed to their disease at months 4, 30 and 18, respectively (Fig. 1a, Supporting Information Fig. S6). While in Patient \#10, the disease progression was associated with the known BTK Cys481Ser and PLCG2 Asp993His mutations ${ }^{28}$, in Patient \#9 and Patient \#17, the previously unreported BTK Gln516Lys and Arg490His mutations appeared to be linked with disease progression (Fig. 1a, Supporting Information Fig. S6). In Patient \#1, two BTK Cys481 mutations emerged at month 34 , when the patient received venetoclax treatment, showed a good response and is currently in remission at month 40. Patient \#2 died of a secondary tumor (colon cancer) with an expanding TP53 mutant clone 27 months after starting ibrutinib therapy. Patient \#6 with a BTK Cys481Ser mutation died of septicemia at month 30 with a stable disease (Fig. 1a, Supporting Information Fig. S6). A BTK Cys481Ser mutation was detected in Patient \#11 at month 24, with documented laboratory progression characterized by a gradual increase of lymphocyte count. Patient \#3 developed two previously unreported BTK (Arg28Ser and Gly164Asp) and two novel PLCG2 (Phe82Ser and Ser1192Gly) mutations by month 18 and is still in clinical remission at month 40 on ibrutinib therapy. Patient \#19 acquired a PLCG2 Arg694His variant (previously unreported in CLL) at month 8 on ibrutinib treatment and died without CLL progression due to a secondary tumor (malignant melanoma) within 2 months.

\section{Monitoring of BTK Cys481Ser and PLCG2 Asp993His mutations using digital droplet PCR}

Twelve and 15 months after the time of the latest sample collection for NGS analysis, Patients \#5 and \#20 also acquired BTK Cys481Ser mutation at months 44 (VAF: $0.03 \%$ ) and 32 (VAF: $10.1 \%$ ) post-ibrutinib, respectively, as identified by ddPCR. These patients are demonstrating laboratory progression at the moment, with permanently increasing lymphocyte count without clinically manifest progression to date. 
(a)

BTK (Chr. Xq22.1)

Richter transformation

CLL progression

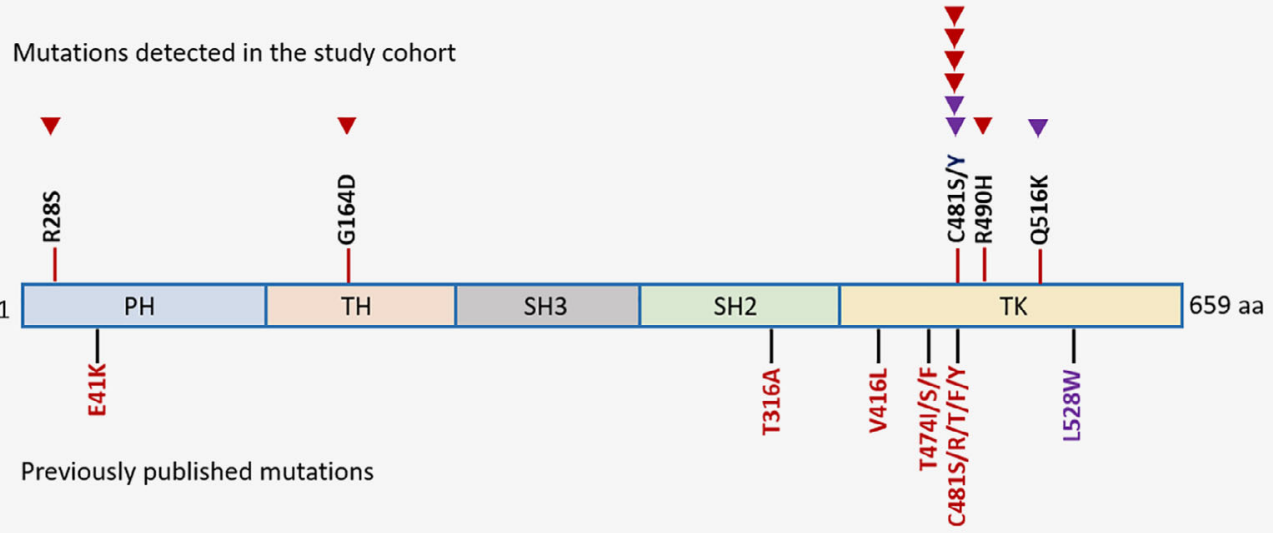

(b)

PLCG2 (Chr. 16q23.3)

Richter transformation

CLL progression

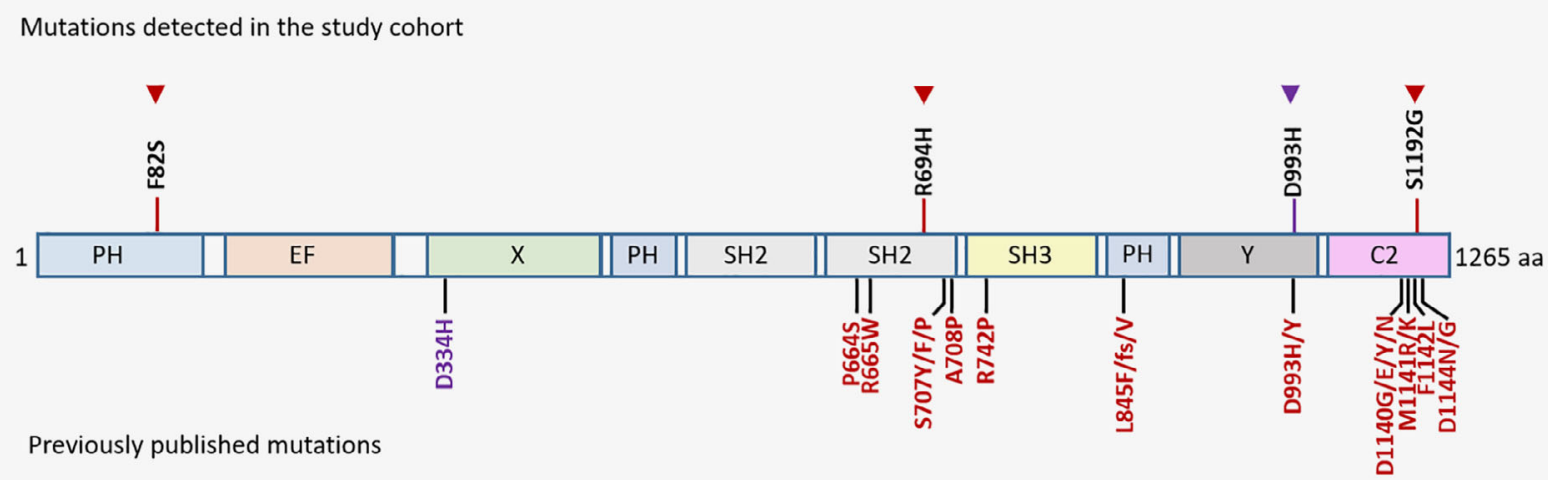

Figure 2. (a) Schematic domain structure of BTK with variants observed in our study cohort and/or identified by previous studies. We observed mutations within the PH, TH and TK domains of the protein. (PH: Pleckstrin homology; TH: Tec homology; SH3/2: Src homology 3/2; TK: Tyrosine kinase). Variants highlighted with red were detected in patients with CLL, while purple variants were observed in patients with Richter's transformation. BTK R28S, G164D, R490H and Q516K represent previously unreported variants, based on the COSMIC database. (b) Schematic domain structure PLCG2 with variants observed in our study cohort and/or identified by previous studies. (EF: EF-hand motifs; X: X domain; SH2/3: Src homology 2/3; Y: Y domain; C2: calcium-binding motif) Variants highlighted with red were detected in patients with CLL, while purple variants were observed in patients with Richter's transformation. PLCG2 F82S and S1192G are previously unreported variants not annotated in COSMIC database. The PLCG2 R694H variant was previously reported in two colon cancer cases (COSM2693625); however, it represents a novel finding in CLL.

Using serial samples and a highly sensitive $(0.01 \%)$ digital droplet PCR (ddPCR) assay, we retrospectively backtracked the BTK/PLCG2 mutations in Patients \#5 and \#20 and in three additional patients (Patients \#1, \#10 and \#11) to determine the earliest time point in the clonal history when the mutations gained selective advantage. The BTK/PLCG2 variants emerged on average 10.5 months (range: 7-15 months) before the clinical relapse with low fractional abundance (Fig. 3). In Patient \#11, laboratory progression developed 10 months after the first detection of the BTK Cys481Ser mutation. In Patients \#1 and \#10, we also demonstrated the reduction of the BTK mutant subclones upon venetoclax treatment (Fig. 3). The extended clonal history of Patient \#10 including spatial mutation profiling was previously published. ${ }^{27}$
Overall, $78 \%$ (7/9) of the patients with BTK mutations showed laboratory or clinical disease progression with these mutations predating relapse by several months (Supporting Information Fig. S6), while no progression occurred without underlying BTK mutations in our cohort. In all three patients with Richter syndrome, the BTK mutations emerged within the first 2 years (4-18 months) of ibrutinib therapy, while patients with CLL progression acquired BTK mutations after a longer exposure to ibrutinib (21-28 months), suggesting that early acquisition of $B T K$ mutations may predict a rapidly progressing disease course.

\section{Discussion}

Ibrutinib has remarkably changed the landscape of CLL therapy by inducing durable responses in patients with previously 

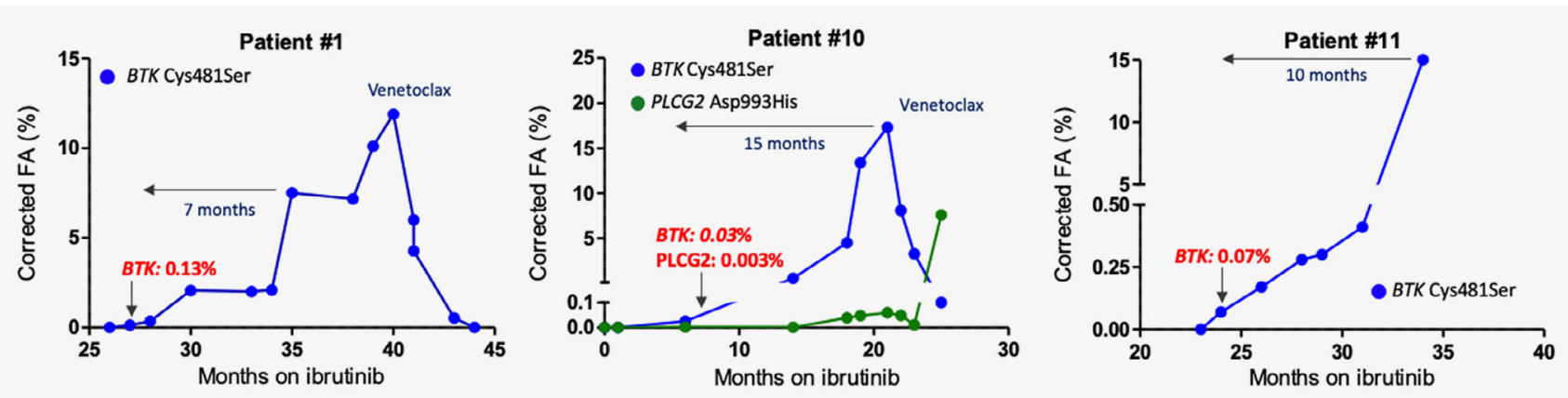

Patient \#5
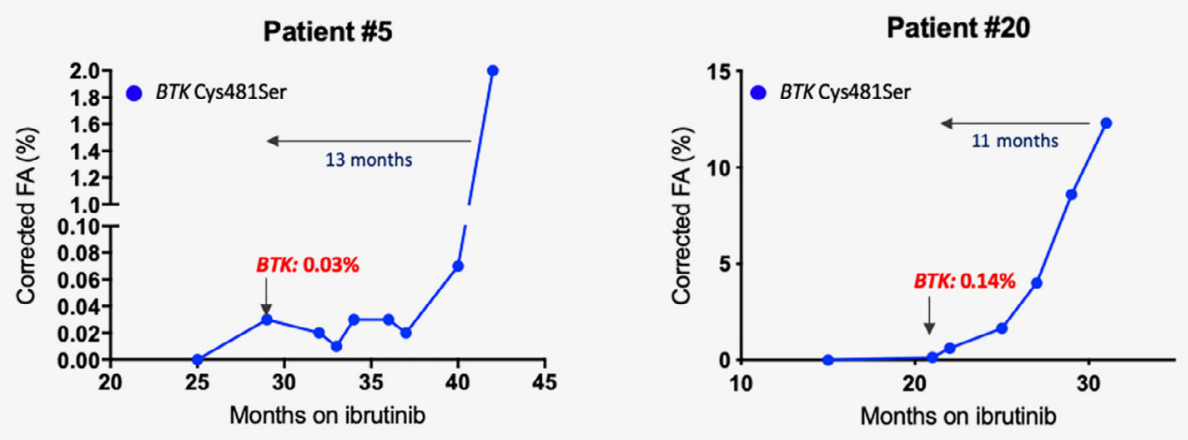

Figure 3. Digital droplet PCR assay was employed to track the BTK Cys481Ser and PLCG2 Asp993His mutations in five patients. The BTK/ PLCG2 variants were detected on average 10.5 months before clinical relapse. For Patients \#1 and \#10, illustrated are the clonal changes of $B T K$ and PLCG2 mutations after venetoclax treatment.

relapsed/refractory disease as well as in high-risk patients harboring TP53 aberrations. ${ }^{13}$ However, the extent and landscape of subclonal changes occurring under the selective pressure of ibrutinib have not completely been dissected yet. Here, we performed an ultra-deep NGS analysis of 30 mutation target genes on a "real-world" patient cohort treated with ibrutinib.

Dynamic clonal selection emerging on the ground of a profound subclonal heterogeneity and commonly conferring convergent evolution was observed in the vast majority of patients. Scrutiny of the clonal trajectories revealed that all individual cases were characterized by unique combinations of mutations as well as different patterns of clonal variegation upon the selective pressure exerted by ibrutinib. In contrast to clonal selection conferred by chemo-immunotherapy, no mutations in the main driver genes involved in pathogenesis of CLL demonstrated selective fitness to ibrutinib. Our study cohort represented a pretreated patient group with up to five lines of standard chemo-immunotherapies, hence the subclonal architecture prior to ibrutinib therapy was reshaped multiple times consecutively. The common presence of subclonal mutations detected in virtually all genes analyzed in our study is in line with a recent observation by Nadeu et al. reporting for the first time that mutations emerging subclonally are more common in CLL as compared to clonal mutations, and the extent of subclonal heterogeneity has an influence on the survival of CLL patients. ${ }^{28}$ This was confirmed and extended by Leeksma et al. documenting increased subclonal heterogeneity and significant enrichment of recurrent gene mutations in chemotherapy refractory patients as compared to treatment naïve cases, in a cohort of 643 patients. $^{29}$

Although in clinical terms, response to ibrutinib appears to be independent of TP53 aberrations, patients with TP53 defects relapse more frequently, suggesting a complex biology behind TP53 deregulation. Indeed, the TP53 mutant subclones observed in our study showed variable scenarios with undergoing clonal expansion or reduction, or elimination of a TP53 mutant subclone coupled with concurrent acquisition of another subclone with different TP53 mutation, indicating great evolutionary capacity and a differential sensitivity of the various TP53 mutations to ibrutinib. Intriguingly, analysis of the subclonal architecture identified a novel alternating pattern of clonal dynamics of BTK and TP53 mutations. The majority of $B T K$ mutations were detectable after reduction or elimination of TP53 mutated subclones and conversely, were never acquired on the ground of persisting or expanding TP53 mutated subclones. Although this finding should be confirmed in larger patient cohorts, one may speculate that elimination of the TP53 mutant subclones may be a prerequisite for the expansion of subclones harboring BTK mutations in those cases where both mutations are present in any time during disease course. Another alternative would be that the prolonged duration of ibrutinib treatment (in our cohort, 2.5-3 years) simply creates niche for subclones 
harboring various $B T K$ mutations by gradually eliminating the TP53 subclones.

With a median follow up time of 36.5 months, 35\% (7/20) of the patients relapsed with a BTK or PLCG2 mutation in our cohort. With our study, we reinforced and extended the central role of BTK and PLCG2 mutations in ibrutinib resistance by identifying novel putative mutation sites in the BTK gene with two previously unreported BTK mutations (Arg490His and Gln516Lys) in two of the patients displaying ibrutinib resistance and rapid disease progression. We also provide evidence that early and sensitive detection of $B T K$ mutations may be predictive of an impending relapse. After the initial discovery of the canonical BTK and PLCG2 mutations underlying ibrutinib resistance by whole exome sequencing studies, ${ }^{14,15}$ additional mutations were described in various domains of these two genes, predominantly detected in single patients with low VAFs. ${ }^{16,17,30-32}$ As in many of the initial studies, only mutation hotspots were analyzed or NGS studies were performed with a relatively low sequencing depth, the clonal complexity of ibrutinib resistance in the context of BTK and PLCG2 mutations may well have been underestimated. Our study and some of the most recent data in the literature suggest that comprehensive deep-sequencing of these driver genes instead of a restrictive analysis of mutation hotspots may be of added clinical value in the future. In addition, genomic profiling of multiple sites, such as peripheral blood, bone marrow and lymph nodes providing distinct microenvironments for parallel clonal evolution should be considered in order to achieve a precise characterization of CLL in individual patients. ${ }^{27}$

\section{Acknowledgements}

This work was funded by the KH17-126718, K_16 \#119950, NVKP_16-1-2016-0004 and NVKP_16-1-2016-0005 grants of the Hungarian National Research, Development and Innovation Office (NKFIH), the Momentum grant (LP-95021) and the János Bolyai Research Scholarship Program $(\mathrm{BO} / 00320 / 18 / 5)$ of the Hungarian Academy of Sciences, the UNKP18-4-SE-62, ÚNKP-18-3-I-SE-48 and EFOP-3.6.3-VEKOP-16-2017-00009 grants of the Ministry of Human Capacities and the Higher Education Institutional Excellence Programme of the Ministry of Human Capacities in Hungary, within the framework of the Molecular Biology Thematic Programme of the Semmelweis University.

\section{Author contributions}

Study concept and design, data analysis and article writing: Gángó $\mathrm{A}$, Alpár $\mathrm{D}$, Mátrai $\mathrm{Z}$ and Bödör C. Bioinformatic analyses: Galik B and Gyenesei A. Experimental work and data analysis: Marosvári D, Kiss R, Fésüs V, Aczél D, Eyüpoglu E, Nagy N, Nagy A, Krizsán S and Reiniger L. Samples collection, management and data analysis: Farkas P, Kozma A, Ádám E, Tasnády S, Réti M and Matolcsy A. Review and final approval of the article: all authors.

\section{References}

1. Baliakas P, Hadzidimitriou A, Sutton LA, et al. Recurrent mutations refine prognosis in chronic lymphocytic leukemia. Leukemia 2015;29:329-36.

2. Landau DA, Carter SL, Stojanov P, et al. Evolution and impact of subclonal mutations in chronic lymphocytic leukemia. Cell 2013;152:714-26.

3. Landau DA, Tausch E, Taylor-Weiner AN, et al. Mutations driving CLL and their evolution in progression and relapse. Nature 2015;526:525-30.

4. Puente XS, Pinyol M, Quesada V, et al. Wholegenome sequencing identifies recurrent mutations in chronic lymphocytic leukaemia. Nature 2011; 475:101-5.

5. Quesada V, Conde L, Villamor N, et al. Exome sequencing identifies recurrent mutations of the splicing factor SF3B1 gene in chronic lymphocytic leukemia. Nat Genet 2011;44:47-52.

6. Schuh A, Becq J, Humphray S, et al. Monitoring chronic lymphocytic leukemia progression by whole genome sequencing reveals heterogeneous clonal evolution patterns. Blood 2012;120: 4191-6.

7. Wang L, Lawrence MS, Wan Y, et al. SF3B1 and other novel cancer genes in chronic lymphocytic leukemia. N Engl J Med 2011;365:2497-506.

8. Malcikova J, Stano-Kozubik K, Tichy B, et al. Detailed analysis of therapy-driven clonal evolution of TP53 mutations in chronic lymphocytic leukemia. Leukemia 2015;29:877-85.

9. Rossi D, Cerri M, Deambrogi C, et al. The prognostic value of TP53 mutations in chronic lymphocytic leukemia is independent of Del17p13: implications for overall survival and chemorefractoriness. Clin Cancer Res 2009;15: 995-1004.

10. Burger JA, Tedeschi A, Barr PM, et al. Ibrutinib as initial therapy for patients with chronic lymphocytic leukemia. N Engl J Med 2015;373:2425-37.

11. Byrd JC, Brown JR, O'Brien S, et al. Ibrutinib versus ofatumumab in previously treated chronic lymphoid leukemia. N Engl J Med 2014;371: 213-23.

12. Farooqui MZ, Valdez J, Martyr S, et al. Ibrutinib for previously untreated and relapsed or refractory chronic lymphocytic leukaemia with TP53 aberrations: a phase 2, single-arm trial. Lancet Oncol 2015;16:169-76

13. Ahn IE, Farooqui MZH, Tian X, et al. Depth and durability of response to ibrutinib in CLL: 5-year follow-up of a phase 2 study. Blood 2018;131: 2357-66.

14. Furman RR, Cheng S, Lu P, et al. Ibrutinib resistance in chronic lymphocytic leukemia. $N$ Engl J Med 2014;370:2352-4.

15. Woyach JA, Furman RR, Liu TM, et al. Resistance mechanisms for the Bruton's tyrosine kinase inhibitor ibrutinib. N Engl J Med 2014;370: 2286-94.

16. Ahn IE, Underbayev C, Albitar A, et al. Clonal evolution leading to ibrutinib resistance in chronic lymphocytic leukemia. Blood 2017;129:1469-79.

17. Maddocks KJ, Ruppert AS, Lozanski G, et al. Etiology of ibrutinib therapy discontinuation and outcomes in patients with chronic lymphocytic leukemia. JAMA Oncol 2015;1:80-7.

18. Woyach JA, Ruppert AS, Guinn D, et al. BTKC481S-mediated resistance to ibrutinib in chronic lymphocytic Leukemia. J Clin Oncol 2017; 35:1437-43.

19. Landau DA, Sun C, Rosebrock D, et al. The evolutionary landscape of chronic lymphocytic leukemia treated with ibrutinib targeted therapy. Nat Commun 2017;8:2185.

20. Jain P, Keating M, Wierda W, et al. Outcomes of patients with chronic lymphocytic leukemia after discontinuing ibrutinib. Blood 2015;125:2062-7.

21. Rosenquist R, Ghia P, Hadzidimitriou A, et al. Immunoglobulin gene sequence analysis in chronic lymphocytic leukemia: updated ERIC recommendations. Leukemia 2017;31:1477-81.

22. Wilm A, Aw PP, Bertrand D, et al. LoFreq: a sequence-quality aware, ultra-sensitive variant caller for uncovering cell-population heterogeneity from high-throughput sequencing datasets. Nucleic Acids Res 2012;40:11189-201.

23. Cingolani P, Platts A, Wang le L, et al. A program for annotating and predicting the effects of single nucleotide polymorphisms, SnpEff: SNPs in the genome of Drosophila melanogaster strain w1118; iso-2; iso-3. Fly 2012;6:80-92.

24. Wang K, Li M, Hakonarson H. ANNOVAR: functional annotation of genetic variants from highthroughput sequencing data. Nucleic Acids Res 2010;38:e164.

25. Bouaoun L, Sonkin D, Ardin M, et al. TP53 variations in human cancers: new lessons from the IARC TP53 database and genomics data. Hum Mutat 2016;37:865-76.

26. Tikkanen T, Leroy B, Fournier JL, et al. Seshat: a web service for accurate annotation, validation, 
and analysis of TP53 variants generated by conventional and next-generation sequencing. Hum Mutat 2018;39:925-33.

27. Kiss R, Alpar D, Gango A, et al. Spatial clonal evolution leading to ibrutinib resistance and disease progression in chronic lymphocytic leukemia. Haematologica 2018;104:e38-41.

28. Nadeu F, Clot G, Delgado J, et al. Clinical impact of the subclonal architecture and mutational complexity in chronic lymphocytic leukemia. Leukemia 2017;32:645-53.

29. Leeksma AC, Taylor J, Wu B, et al. Clonal diversity predicts adverse outcome in chronic lymphocytic leukemia. Leukemia 2018;33:390-402.

30. Burger JA, Landau DA, Taylor-Weiner A, et al. Clonal evolution in patients with chronic lymphocytic leukaemia developing resistance to BTK inhibition. Nat Commun 2016;7:11589.
31. Jones D, Woyach JA, Zhao W, et al. PLCG2 C2 domain mutations co-occur with BTK and PLCG2 resistance mutations in chronic lymphocytic leukemia undergoing ibrutinib treatment. Leukemia 2017;31:1645-7.

32. Sharma S, Galanina N, Guo A, et al. Identification of a structurally novel BTK mutation that drives ibrutinib resistance in CLL. Oncotarget 2016;7: 68833-41. 